\title{
On 3D Reconstruction of Porous Media by Using Spatial Correlation Functions
}

\author{
G.A. Papakostas ${ }^{1,2, *}$, J.W. Nolan ${ }^{2}$, N. $\operatorname{Vordos}^{2}$, D. Gkika ${ }^{2}$, M. E. Kainourgiakis ${ }^{3}$ and A. C. Mitropoulos ${ }^{2}$ \\ ${ }^{1}$ Human Machines Interaction (HMI) Laboratory, Department of Computer and Informatics Engineering, Eastern Macedonia \\ and Thrace Institute of Technology, GR-65404 St. Lukas, Kavala, Greece \\ ${ }^{2}$ Hepheastus Laboratory, Department of Petroleum and Mechanical Engineering, Eastern Macedonia and Thrace Institute of Technology, \\ GR-65404, St. Lucas, Kavala, Greece \\ ${ }^{3}$ Environmental Research Laboratory, NCSR 'Demokritos', 15310 Athens, Greece
}

Received 31 October 2014; Accepted 9 November 2015

\begin{abstract}
The challenging process of 3D porous media reconstruction from a single $2 \mathrm{D}$ image is investigated in this paper. The reconstruction of the 3D model is based on the statistical information derived from a $2 \mathrm{D}$ thin image of the material, by applying a spatial correlation function. For the first time, this paper reviews the commonly used auto-correlation functions for material characterization and discusses their properties making them useful for 3D porous media reconstruction. A set of experiments is conducted in order to analyze the reconstruction capabilities of the studied correlation functions, while some useful conclusions are drawn. Finally, by taking into account the reconstruction performance of the existed correlation functions, some desirable properties that need to be satisfied by an ideal correlation function towards the improvement of the reconstruction accuracy are determined.
\end{abstract}

Keywords: 3D reconstruction, porous media, material characterization, correlation function.

\section{Introduction}

Recently, there is an increased interest in using porous media in many disciplines of the engineering life such as petroleum [1] and chemical industries [2], geosciences [3], biology [4] etc. This broad usage of porous media has brought to the foreground the needs for accurate analysis of the macroscopic and flow behaviour of these materials, stemming from their pore structure. The accurate determination of the pore structure permits the measurement of the transport properties of the materials such as diffusivity, permeability, conductivity etc.

A common practice in determining the geometry and topology of the pore space of porous media is through the construction of a 3D model of the true material. Such a method, called $3 D$ reconstruction, uses information from the original material and aims to reconstruct the material by providing an approximate model of it. A typical reconstruction algorithm uses information extracted from a single $2 \mathrm{D}$ thin image slice of the original material, instead of multiple slices.

Since the introduction of the first reconstruction methodology by Quiblier [5], several techniques have been proposed through the years [6-13], having as primary goal to improve the reconstruction accuracy. The reconstruction accuracy is mainly affected by the way the microstructure of the true porous material is captured, as well as the method used to translate this information to a $3 \mathrm{D}$ model.

* E-mail address: gpapak@teikav.edu.gr ISSN: 1791-2377 @ 2015 Kavala Institute of Technology. All rights reserved.
As far as the description of the porous space is concerned, a specific spatial correlation function is commonly used. The spatial correlation functions [14] such as auto-correlation functions have proved to be good descriptors of the porous space morphology, while their simplicity accelerates the overall reconstruction procedure. Although several auto-correlation functions have been used in $3 \mathrm{D}$ porous media reconstruction, some of them sharing the same properties, there is no study regarding the efficiency each of them has, in terms of reconstruction accuracy.

The contribution of this paper is threefold. Firstly, it reviews the spatial correlation functions mostly used in $3 \mathrm{D}$ reconstruction of porous media, by describing their properties. Secondly, this paper evaluates the reconstruction performance of the examined correlation functions, as a part of the same reconstruction algorithms and under identical configuration settings. Thirdly, this work discusses the suitability of each correlation function in solving the reconstruction problem, by resulting to the determination of the ideal correlation function that need to be developed in order to achieve the highest reconstruction accuracy.

This paper is organized as follows: Section 2, briefly describes the main principles of porous media 3D reconstruction with emphasis to a specific algorithm applied to all experiments. Section 3, reviews the most used spatial correlation functions and summarizes their properties in describing the pore space of the materials. Section 4, evaluates the reconstruction performance of the correlation functions and illustrates reconstructed 3D models in each case. Section 5, discusses the main experimental outcomes and revisits the practical needs that should be satisfied by an ideal correlation function. Finally, Section 6 concludes the 
previous analysis and puts forward the future research directions that need to be addressed.

\section{3D Reconstruction of Porous Media}

The study of the macroscopic behaviour of the porous media requires the determination of the geometry and topology of the pore space. This can be achieved by examining the $3 \mathrm{D}$ representation of the porous media that can be derived by using specialized microscopes (TEM, GEM, etc.). However, the construction of the $3 \mathrm{D}$ representation of the porous media using multiple $2 \mathrm{D}$ cuts is time consuming, whereas the cost of the microscopes is very high.

In order to overcome the aforementioned obstacles in deriving the $3 \mathrm{D}$ porous media structure, several methods have been proposed for reconstructing an approximated 3D model of the porous media using a single $2 \mathrm{D}$ thin slice of the material. These methods are mainly divided into two categories: (1) statistical/stochastic methods that apply linear and non-linear filters to an uncorrelated Gaussian distribution [5-8, 11] and (2) optimization-based methods that tackle the reconstruction process as an optimization problem subject to some objectives [9-10]. While the latter methods give more accurate results in comparison with the former ones, their high computation time makes them less attractive.

The aforementioned two categories of $3 \mathrm{D}$ reconstruction methods share the same measure to encode the porous space of the 2D cross section, through multiple-point autocorrelation functions. These functions are capable to describe statistically the microstructures of the 2D slice of porous media and thus can be used to construct 3D models of the same statistical properties.

In this study, a simplified version [7] of the reconstruction algorithm of Quiblier [5] has been adopted and used in the hereafter experiments. According to this method [7] the two-point auto-correlation function of the 2D image having intensity function $I(x, y)$ and $M \times N$ pixels size, is computed by applying the following Eq.(1):

$S(x, y)=\sum_{i=1}^{M} \sum_{j=1}^{N} \frac{I(i, j) \times I(i+x, j+y)}{M \times N}$

It is worth noting that the method of periodic boundaries is applied for the coordinates lying outside the image's dimensions.

The two-point auto-correlation function of Eq.(1) for a distance $r=\sqrt{x^{2}+y^{2}}$ is defined as follows:

$$
S(r)=\frac{1}{2 r+1} \sum_{l=0}^{2 r} S\left(r, \frac{\pi l}{4 r}\right)
$$

where the form of $S(r, \theta)$ in polar coordinates with $r=r \cos \theta, r=r \sin \theta$, is derived by interpolating Eq.(1).

Initially, a Gaussian distributed noise $G(x, y, z)$, properly transformed to uniform and independent by applying the Box-Muller method, is considered as the starting form of the 3D model of the material.
Moreover, the auto-correlation function of the $3 \mathrm{D}$ model statistically is statistically related with the auto-correlation function of the 2D image Eq.(2), according to:

$$
F(r)=F(x, y, z)=\frac{S(r)-(S(0))^{2}}{S(0)-(S(0))^{2}}
$$

with $r=\sqrt{x^{2}+y^{2}+z^{2}}$ being the distance between porous points in the three-dimensional coordinate space of the material model.

The resulted 3D model can be derived by convolving signal $G(x, y, z)$ with the $F(x, y, z)$ filter Eq.(4) in a simplified way, by avoiding the solving of several systems of nonlinear equations towards the computation of the filter coefficients of the convolved filter.

$$
R(x, y, z)=\sum_{i=0}^{30} \sum_{j=0}^{30} \sum_{k=0}^{30} N(x+i, y+j, z+k) \times F(i, j, k)
$$

The resulted 3D model (Eq.(4)) of the porous media, has intensity values lying to the real numbers space and thus it should be transformed to binary in order to indicate the porous space. For this reason, a thresholding (binarization) algorithm need to be applied, by also satisfying the additional requirement of the equal porosity (number of pixels belonging to the porous space) between the reconstructed $3 \mathrm{D}$ model and the original three-dimensional material image.

Finally, during the verification stage the auto-correlation function of the derived 3D model should be compared with that function used as the convolved filter, so that a reconstruction error could be defined.

\section{Pore Space Description}

The 3D reconstruction of a porous media, as described in the previous section, is mainly based on the appropriate description of the pore space of the $2 \mathrm{D}$ thin image and the assumption the 3D model shows the same statistical behavior with that of the $2 \mathrm{D}$ sample image. Towards this direction, a common practice is to calculate the first two moments of the 2D image namely porosity $(\varepsilon)$ and autocorrelation $\left(\mathrm{R}_{\mathrm{z}}\right)$, which for the case of stationary and isotropic materials are the same for any dimension.

It is worth noting that a spatial correlation function measures the probability of some points to lie in the same space (material or pore for two-phase materials). For example the two-point correlation function measures the probability two points in a specified distance lie both in the same phase.

Based on the aforementioned notations the following definition for the porous materials characterization (3D material reconstruction) problem can be stated:

Definition 1: Find the $3 D$ pore space geometry and topology (model) that shows the same high order statistics (porosity and two-point, three-point, multiple-point correlation functions) with any $2 D$ cross-section of the same material. 
Assuming a 2D thin cross-section of a porous media, the following phase function is defined for a binary material description:

$Z(\mathbf{x})= \begin{cases}1, & \text { if } \mathbf{x} \in \text { porespace } \\ 0, & \text { if } \mathbf{x} \in \text { material }\end{cases}$

where $\mathbf{x}$ is the vector of coordinates of each crosssection's pixel.

Based on the above definition the first two statistical moments of the 2D material slice are defined as:

Porosity $\varepsilon=\langle Z(\mathbf{x})\rangle$

and

Two-point
Auto-correlation $\quad R_{z}(\mathbf{u})=\frac{\langle(Z(\mathbf{x})-\varepsilon) \cdot(Z(\mathbf{x}+\mathbf{u})-\varepsilon)\rangle}{\varepsilon-\varepsilon^{2}}$

where \langle\rangle is the average operator.

A comparison of the two auto-correlation functions of Eq.(1) and Eq.(7), yields to the conclusion that although these quantities are very similar they are not identical. In fact a great number of auto-correlation functions that slightly differ are used for 3D material reconstruction.

Since it is not clear which function is more appropriate to reconstruct the $3 \mathrm{D}$ material with minimum error, there is a need to compare the reconstruction performance of the commonly used auto-correlation functions, under the same configuration.

For this purpose, an in-depth study of the autocorrelation functions, widely used in the literature for material characterization, has taken place herein and the most representative functions have been selected for the experimental section that follows.

It should be mentioned that although the definition of the auto-correlation functions differ in respect to the used nomenclature, a special effort towards the unified definition of the functions has also been made. Moreover, this study is restricted only to two-point autocorrelation functions, since higher order functions significantly increase the overall computation time.

The following two point auto-correlation functions are commonly used in the literature and will be compared in reconstructing the $3 \mathrm{D}$ material model using the methodology of section 2 .

$$
\begin{aligned}
& R_{z}^{1}=\frac{\langle(Z(\mathbf{x})-\varepsilon) \cdot(Z(\mathbf{x}+\mathbf{u})-\varepsilon)\rangle}{\left\langle(Z(\mathbf{x})-\varepsilon)^{2}\right\rangle} \\
& R_{z}^{2}=\frac{\langle(Z(\mathbf{x})-\varepsilon) \cdot(Z(\mathbf{x}+\mathbf{u})-\varepsilon)\rangle}{\left(\varepsilon-\varepsilon^{2}\right)} \\
& R_{z}^{3}=\frac{\langle Z(\mathbf{x}) \cdot Z(\mathbf{x}+\mathbf{u})\rangle-\varepsilon^{2}}{\left(\varepsilon-\varepsilon^{2}\right)}
\end{aligned}
$$

It is worth noting that the above two point autocorrelation functions are quite similar and they have been used in several papers in the last thirty years. More precisely, $R_{z}^{1}$ was firstly proposed by Quiblier [5] and used also in [15], $R_{z}^{2}$ was proposed by Adler et al. [6] and used in $[8,11,13,14]$, while $R_{z}^{3}$ was used in $[7,16]$.

\section{Experiments}

A set of experiments has been conducted in order to study the reconstruction capabilities of the previously described two point auto-correlation functions. As a matter of fact, specific software has been developed in MATLAB, while all experiments were executed in an Intel i5 $3.3 \mathrm{GHz}$ PC with 8GB RAM. Moreover, a 2D thin binary section of a random sphere pack $(\varepsilon=0.4334)$ (Fig.1) [11] having $100 \times 100$ pixels size was selected to participate in all reconstruction experiments.

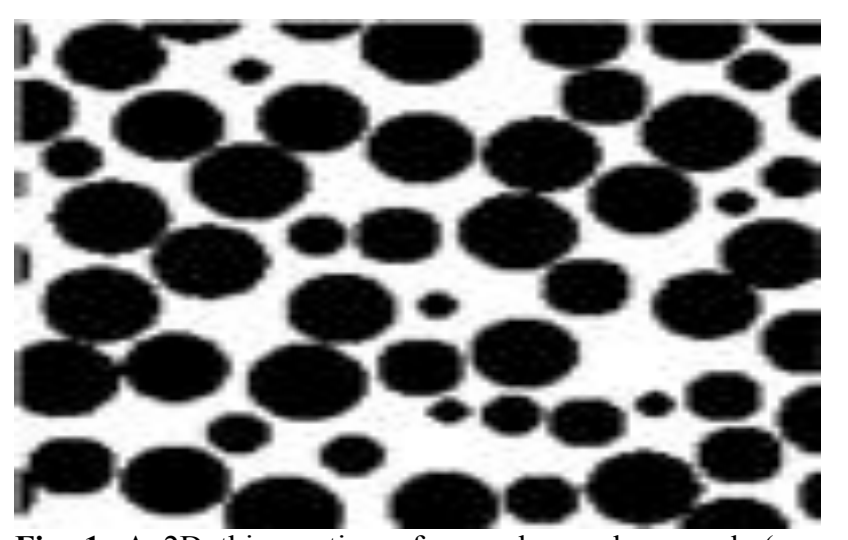

Fig. 1. A $2 \mathrm{D}$ thin section of a random sphere pack $(\varepsilon=$ $0.4334)$.

The $2 \mathrm{D}$ thin section of a random sphere pack (Fig. 1) is used as a benchmark material in order to study the reconstruction performance of the three auto-correlation functions. In the following Fig.(2)-(4), the reconstruction results in each case are illustrated.

More precisely, these figures depict the first cut of the $3 \mathrm{D}$ reconstructed model of the material, the auto-correlation function versus the translation parameter for both the initial 2D section and the first reconstructed section, and finally the $3 \mathrm{D}$ reconstructed material.

By examining the reconstruction results of the three auto-correlation functions, can be concluded that the $R_{z}^{1}$ gives the most accurate $3 \mathrm{D}$ model, since the autocorrelation function of the reconstructed section is close to that of the initial section. This is also justified by the porosity value of the reconstructed section $(\varepsilon=0.4315)$, which is very close to the porosity $(\varepsilon=0.4334)$ of the initial $2 \mathrm{D}$ section.

Furthermore, the reconstruction results of $R_{z}^{2}$ and $R_{z}^{3}$ seems to be identical, since the plots of their auto-correlation functions differ slightly. However, if one compares the porosities of the reconstructed and the initial sections in these cases, it can be concluded that the $R_{z}^{2}$ function yields a more accurate porosity value equal to 0.4655 , while the $R_{z}^{3}$ function gives 0.4933 . 


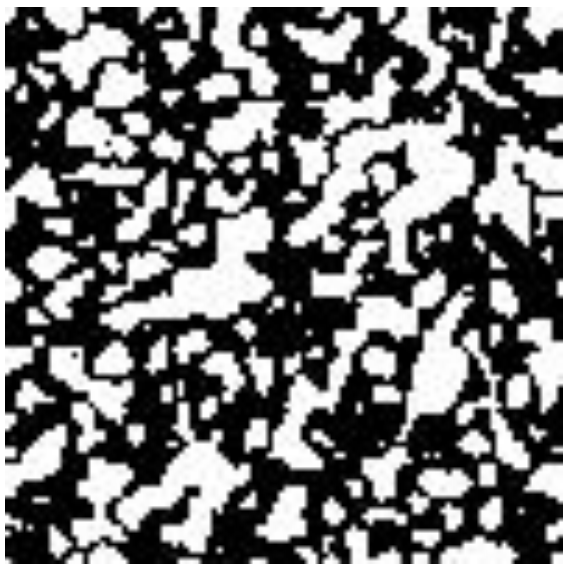

(a)

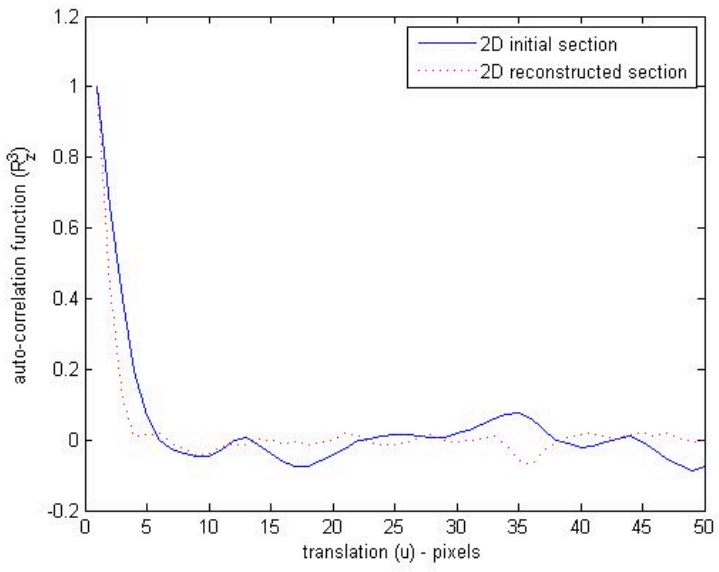

(b)

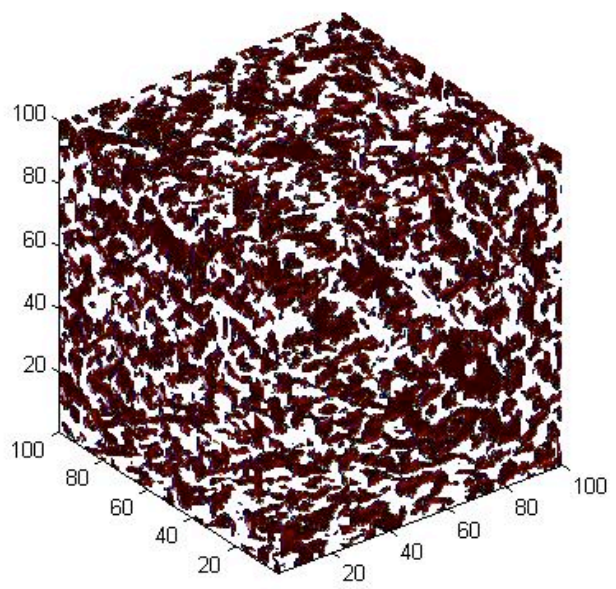

(c)

Fig. 2. Reconstruction results using the $R_{z}^{1}$ auto-correlation function, (a) $2 \mathrm{D}$ reconstructed section $(\varepsilon=0.4315)$, (b) and (c) 3D reconstructed material.

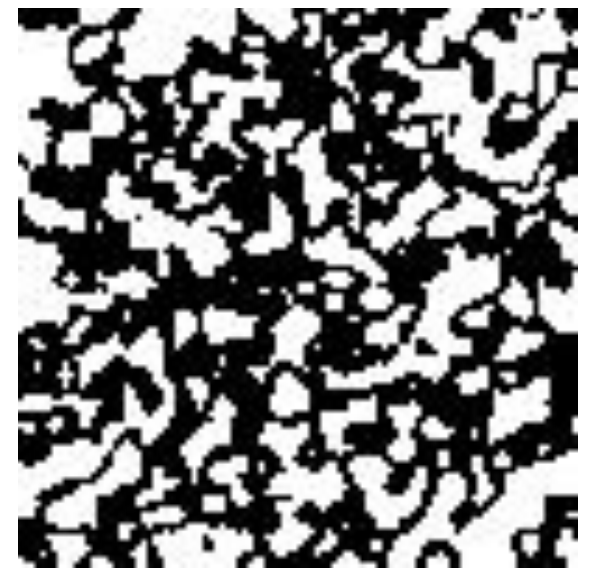

(a)

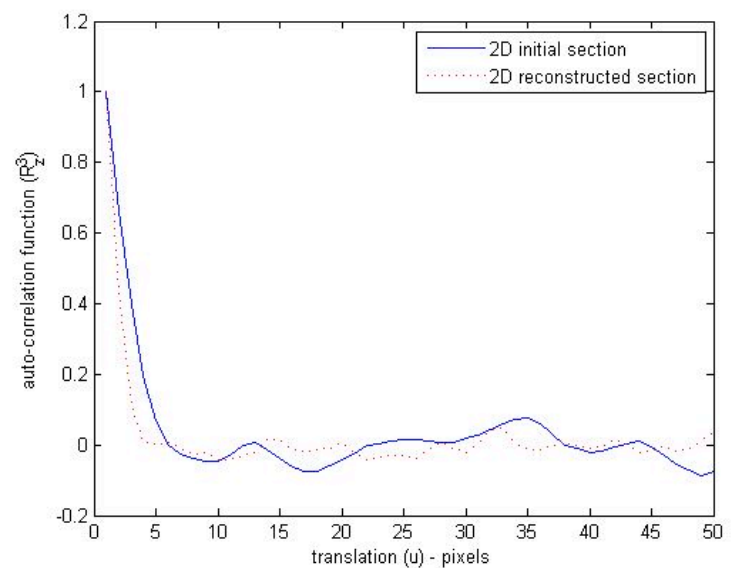

(b)

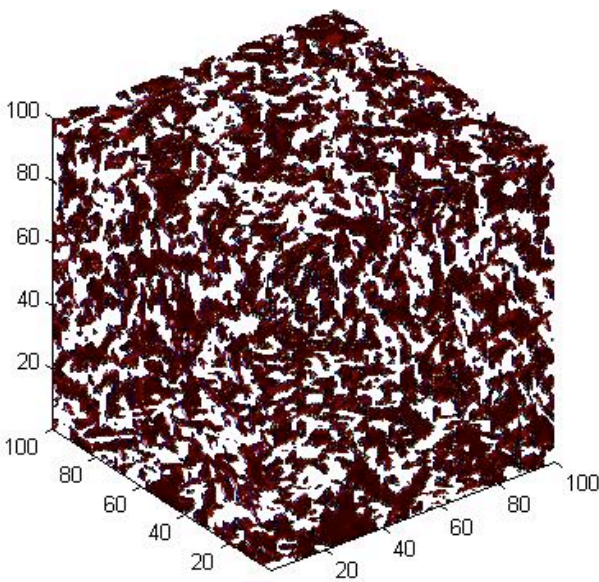

(c)

Fig. 3. Reconstruction results using the $R_{z}^{2}$ auto-correlation function, (a) $2 \mathrm{D}$ reconstructed section $(\varepsilon=0.4655)$, (b) and (c) $3 \mathrm{D}$ reconstructed material. 
(a)
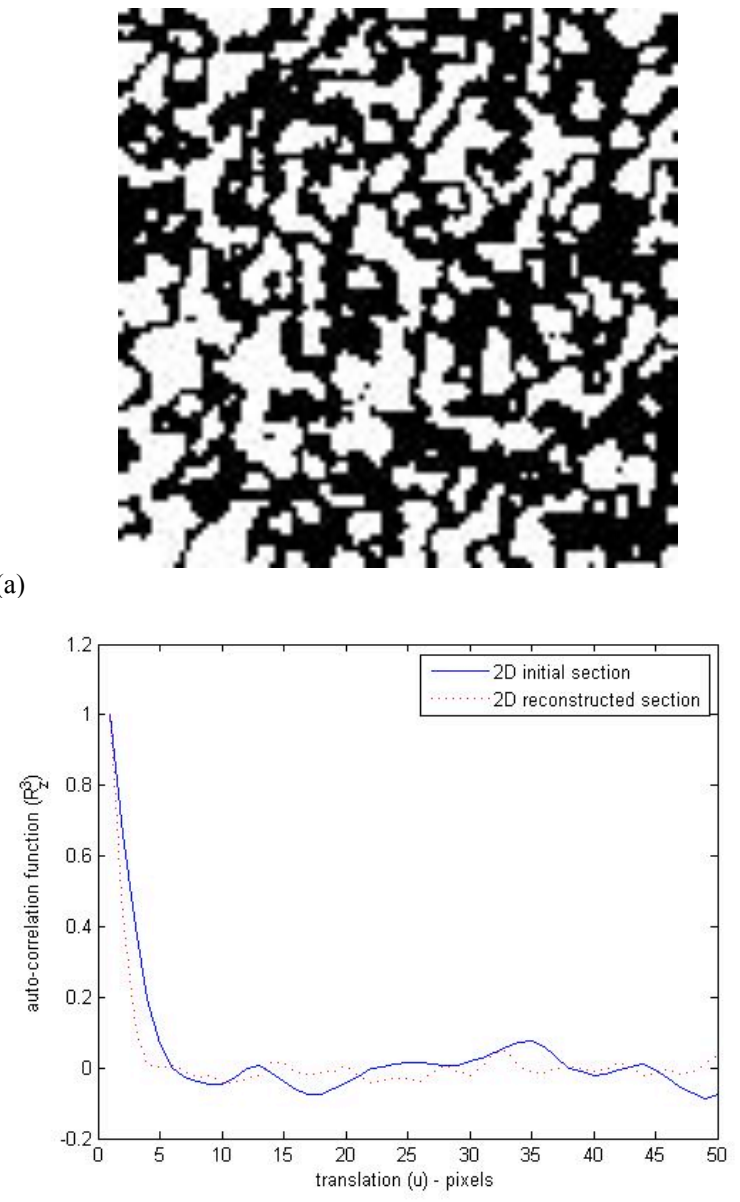

(b)

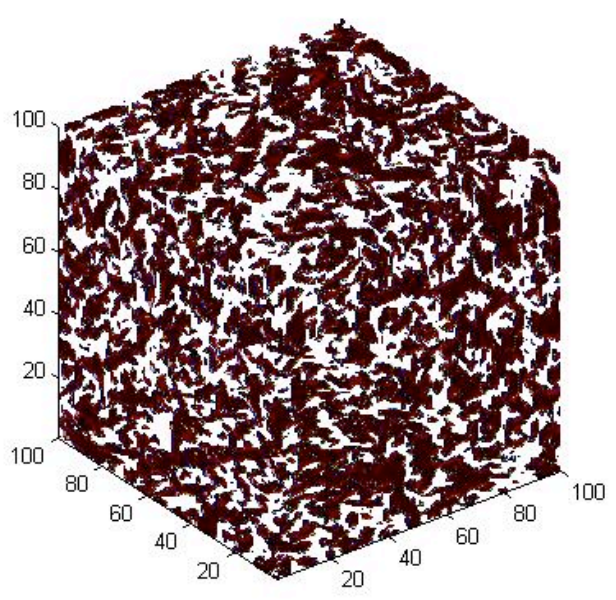

(c)

Fig. 4. Reconstruction results using the $R_{z}^{3}$ auto-correlation function, (a) $2 \mathrm{D}$ reconstructed section $(\varepsilon=0.4933)$, (b) and (c) $3 \mathrm{D}$ reconstructed material.
G.A. Papakostas, J.W. Nolan, N. Vordos, D. Gkika, M. E. Kainourgiakis and A. C. Mitropoulos/

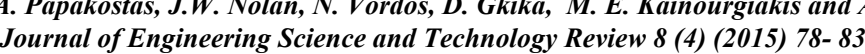

\section{Discussion}

Apart from the comparison results of the auto-correlation functions under investigation, regarding their appropriateness in reconstructing the $3 \mathrm{D}$ material model by using a single $2 \mathrm{D}$ thin section, some very useful outcomes are derived.

A careful inspection of the 3D reconstructed material for each case, leads us to the conclusion that there are different $3 \mathrm{D}$ structures showing almost the same porosity and autocorrelation functions. This means that the problem of finding a 3D structure having the same first two statistical orders (porosity and two point auto-correlation function) defined in section 3 , does not have a unique solution.

In order to reduce the space of the candidate models that fit with the statistical properties of the $2 \mathrm{D}$ thin section, the used information from the $2 \mathrm{D}$ thin section in the reconstruction process needs to be increased. Ideally, it is desired to use an auto-correlation function that describes higher order statistics of the 2D section, in order to capture as much as possible information.

This can be possibly achieved by applying multiple-point auto-correlation functions or by constructing novel functions that take into account the distribution of specific micropatterns on the surface of the 2D thin section. However, in this direction, one has to address the increased computation time caused by the usage of more complicated function definitions.

\section{Conclusions}

The previous sections discussed the role of the spatial autocorrelation functions in $3 \mathrm{D}$ reconstruction of porous media by using a single $2 \mathrm{D}$ thin material section. The most commonly used auto-correlation functions from the literature were described in a first step and their reconstruction performance was analysed in a next step.

Apart from the outperformance of the $R_{z}^{1}$ function, the experiments showed that the problem of $3 \mathrm{D}$ reconstruction using the first two statistical moments of the 2D thin section does not have a unique solution.

The usage of multiple-point autocorrelation functions that describe the relations of more points of the material could be a solution to this problem. Moreover, the development of more descriptive functions enclosing higher order statistics can guarantee the uniqueness of the derived $3 \mathrm{D}$ model subject to a predefined $2 \mathrm{D}$ thin material section.

\section{Acknowledgements}

This work is supported and funded by Thalis "NANOCAPILLARY MIS 375233" research project of the Greek Ministry of National Education and Religious Affairs.

\section{References}

1. J.E. Warren, H.S. Price, "Flow in heterogeneous porous media", Society of Petroleum Engineers Journal, vol. 1, no. 3, pp. 153169, 1961.

2. E.P. Favvas, E.P. Kouvelos, G.E. Romanos, G.I. Pilatos, A.C. Mitropoulos, N.K. Kanellopoulos, "Characterization of highly selective microporous carbon hollow fiber membranes prepared from a commercial co-polyimide precursor", Journal of Porous Materials, vol. 15, no. 6, pp. 625-633, 2008.
3. P.E. Gretener, "Fluid pressure in porous media - Its importance in geology: a review", Bulletin of Canadian Petroleum Geology, vol. 17 , no. 3, pp. 255-295, 1969.

4. A.-R.A. Khaled, K. Vafai, "The role of porous media in modeling flow and heat transfer in biological tissues", International Journal of Heat and Mass Transfer, vol. 46, no. 26, pp. 4989-5003, 2003. 


\section{G.A. Papakostas, J.W. Nolan, N. Vordos, D. Gkika, M. E. Kainourgiakis and A. C. Mitropoulos/}

Journal of Engineering Science and Technology Review 8 (4) (2015) 78- 83

5. J.A. Quiblier, "A new three-dimensional modelling technique for studying porous media", Journal of Colloid and Interface Science, vol. 98, no. 1, pp. 1984.

6. P.M. Adler, C.G. Jacquin and J.A. Quiblier, "Flow in simulated porous media", Int. J. Multiphase Flow, vol. 16, no. 4, pp. 691712, 1990.

7. D.P. Bentz and N.S. Martys, "Hydraulic radius and transport in reconstructed model three-dimensional porous media", Transport in Porous Media, vol. 17, pp. 221-238, 1994.

8. Z.R. Liang, C.P. Fernandes, F.S. Magnani, P.C. Philippi, "A reconstruction technique for three-dimensional porous media using image analysis and Fourier transforms", Journal of Petroleum Science and Engineering, vol. 21, no. 3-4, pp. 273-283, 1998.

9. C.L.Y. Yeong and S. Torquato, "Reconstructing random media", Physical Review E, vol. 57, no. 1, pp. 495-506, 1998.

10. C.L.Y. Yeong and S. Torquato, "Reconstructing random media II. Three-dimensional media from two-dimensional cuts", Review E, vol. 58, no. 1, pp. 224-233, 1998.

11. M.E. Kainourgiakis, E.S. Kikkinides, A.K. Stubos, "Diffusion and flow in porous domains constructed using process-based and stochastic techniques", Journal of Porous Materials, vol. 9, no. 2, pp. 141-154, 2002.
12. Z. Xu, Q. Teng, X. He and Z. Li, “A reconstruction method for three-dimensional pore space using multiple-point geology statistic based on statistical pattern recognition and microstructure characterization", International Journal for Numerical and Analytical Methods in Geomechanics, vol. 37, no. 1, pp. 97-110, 2013.

13. Karthik K. Bodla, Suresh V. Garimella, Jayathi Y. Murthy, "3D reconstruction and design of porous media from thin sections", International Journal of Heat and Mass Transfer, vol. 73, pp. 250264, 2014.

14. James G. Berryman, "Measurement of spatial correlation functions using image processing techniques", J. Appl. Phys., vol. 57, no. 1, pp. 2374-2384, 1985.

15. S.C. Blair, P.A. Berge, and J.G. Berryman, "Using two-point correlation functions to characterize microgeometry and estimate permeabilities of sandstones and porous glass", Journal of Geophysical Research, vol. 101, no. B9, pp. 20359-20375, 1996.

16. E.S. Kikkinides, M.E. Kainourgiakis, K.L. Stefanopoulos, A.C. Mitropoulos, A.K. Stubos, N.K. Kanellopoulos, "Combination of small angle scattering and three-dimensional stochastic reconstruction for the study of adsorption-desorption processes in Vycor porous glass", Journal of Chemical Physics, vol. 112, no. 22, pp. 9881-9887, 2000. 